\section{Regional monitoring plan for the detection of allergens in food from Campania Region. First year monitoring results}

\author{
Rosa Gagliardi, Loredana Biondi, \\ Roberta Pellicanò, Vincenzo Caligiuri, \\ Donatella Nava
}

Istituto Zooprofilattico Sperimentale del Mezzogiorno - Sezione di Portici (NA), Italy

\begin{abstract}
Food allergens are substances able to induce an abnormal immunological response in sensitive individuals. The presence of allergens in food must be reported in tables (Directive 2003/89/EC). In this study we report the data of a monitoring plan carried out in the Campania Region during the 2012 for the detection of allergens (ovoalbumine and $\beta$-lattoglobulin) in food of different origin. The analisys were performed by means of ELISA assays. The percentage of analyzes with the presence of allegens not declared on the label is $4.3 \%$, out of a total of 208 analyzes. It is therefore important to continue monitoring activities by the competent Authorities.
\end{abstract}

\section{Introduzione}

Negli ultimi anni le malattie allergiche, in particolare quelle alimentari, hanno mostrato un trend in crescita, sebbene dall'esame della letteratura non siano disponibili dati che consentano di fare una stima precisa di questo fenomeno (Bush and Hefle, 1996). Per contro, studi recenti, consentono di collocarne la stima approssimativa al $3 \%$ della popolazione mondiale. Per le allergie alimentari non sono disponibili né immunoterapie specifiche né terapie farmacologiche 0 profilassi preventive; l'unico rimedio possibile è evitare di ingerire l'alimento coinvolto (Madsen, 2005; 0sterballe et al., 2005). La Direttiva 2003/89/CE e il Decreto Legislativo n.114 del 8/02/2006 (Commissione Europea, 2003; Repubblica Italiana, 2006) et s.m.i., hanno posto come obiettivo principale la tutela della salute di quella parte della popolazione di soggetti con sensibilità nota nei confronti degli allergeni di origine alimentare (Roehr et al., 2004), definendo l'elenco delle sostanze allergeniche la cui presenza deve essere obbligatoriamente dichiarata in etichetta. Sulla base delle indicazioni citate dalla Direttiva 2003/89/CE (Commissione Europea, 2003), la Regione Campania, nell'ambito del Piano Regionale
Integrato dei Controlli Ufficiali in materia di alimenti, mangimi, sanità e benessere animale e sanità delle piante (P.R.I.) 2011-2014 DGRG 377/11 (Regione Campania, 2011), ha attivato uno specifico Piano di Monitoraggio Regionale per la Ricerca degli Allergeni negli Alimenti, con la finalità di salvaguardare la salute dei soggetti affetti da allergie alimentari e per monitorare l'eventuale presenza di non conformità delle etichette. In base a questo Piano, sono stati sottoposti ad analisi, per la ricerca delle ovoalbumine e della $\beta$-lattoglobulina, i seguenti prodotti: alimenti prima infanzia a base di carne, alimenti destinati ad un'alimentazione particolare non a base di prodotti di origine animale, carni macinate 0 preparazioni di carne, omogeneizzati di carne, piatti pronti non a base di prodotti di origine animale (Taylor et al., 1999; Zuberbier et al., 2004).

\section{Materiali e Metodi}

I campioni sono stati prelevati dai Servizi Veterinari e dai Servizi di Igiene degli Alimenti e della Nutrizione dei Dipartimenti di Prevenzione (SIAN) Regionali ai sensi del DPR $327 / 80$, presso esercizi di vendita e nei depositi all'ingrosso. I Servizi veterinari hanno campionato prodotti di origine animale, mentre i SIAN matrici prive di prodotti di origine animale. Sono stati prelevati 107 campioni sui quali sono state condotte 208 indagini analitiche, $n^{\circ} 105$ per la ricerca della $\beta$-lattoglobulina e n. 103 per la ricerca delle ovoalbumine (Tabella 1); la tipologia delle matrici esaminate è riportata in Tabella 2. Per la rilevazione degli allergeni nelle matrici alimentari analizzate, sono stati utilizzati i kit diagnostici immunoenzimatici ELISA di tipo sandwich diretto, Ridascreen ${ }^{\circledR F}$ Fast $\beta$ Lactoglobulin per la rilevazione della $\beta$-lattoglobulina e Ridascreen ${ }^{\circledR}$ Fast Ei/Egg Protein per le proteine dell'uovo, entrambi prodotti dalla ditta R-Biopharm AG. La preparazione dei campioni e l'allestimento del saggio immunoenzimatico ELISA sono stati effettuati secondo le istruzioni della ditta produttrice dei kit. Le procedure analitiche di estrazione degli allergeni, sono le stesse per entrambi i kits: 1 gr di campione viene prelevato e pesato. L'estrazione è eseguita con l'aggiunta di $20 \mathrm{~mL}$ di buffer di estrazione, specifici per tipologia di prova e forniti dal kit in uso. Successivamente si esegue l'omogeneizzazione in Stomaher (Lab System-Stomacher ®80, Biomaster). Il campione viene lasciato per $10^{\prime}$ a $60^{\circ} \mathrm{C}$ e filtrato con flitro- Whatman ${ }^{\circledR} 41 ø 90$. Una aliquota del surnatante è sottoposta all'analisi immunoenzimatica ELISA.Per la valutazione dei risultati è stato utilizzato il software RIDA®SOFT Win, fornito adal produttore dei kit. I calcoli sono stati eseguiti impiegando la funzione di spline cubica. La lettura è stata effettua-
Correspondence: Rosa Gagliardi, Istituto Zooprofilattico Sperimentale del Mezzogiorno, Via Salute 2, 80055 Portici (NA), Italy. Tel. +39.081 .7865322 - Fax: +39.081 .7865342$

E-mail: rosa.gagliardi@cert.izsmportici.it

Key words: Allergeni, Ovoalbumine, $\beta$-lattoglobulina, ELISA.

Received for publication: 10 May 2013.

Revision received: 5 September 2013.

Accepted for publication: 5 September 2013.

This work is licensed under a Creative Commons Attribution 3.0 License (by-nc 3.0).

CCopyright R. Gagliardi et al., 2014

Licensee PAGEPress, Italy

Italian Journal of Food Safety 2014; 3:1661

doi:10.4081/ijfs.2014.1661

ta a $450 \mathrm{~nm}$, mediante spettrofotometro Microplater Reader mod.680, BIO-RAD. Per entrambi i metodi il campo di misura è dato dalle concentrazioni degli standards forniti dal kit, ovvero 0 ppm - 0,5 ppm - 1,5 ppm - 4,5 ppm - e 13,5ppm, dai quali si ottiene una curva standard di taratura. Sono stati considerati non conformi i campioni che superavano il limite di rilevabilità di $0,5 \mathrm{ppm}$.

\section{Risultati}

Delle 208 analisi effettuate, 9 (4,3\%) son risultate non conformi; 6 (2,9\%) sono risultate non conformi per la ricerca delle ovoalbumine e $3(1,4 \%)$ per la ricerca della $\beta$-lattoglobulina. In un campione è stata rilevata una non conformità per entrambi gli allergeni. Per quanto attiene la tipologia di matrici analizzate, tutte le non conformità sono state rilevate nei prodotti a base di carne: per l'ovoalbumina 3 sono relative a salsicce suine, 1 ad hamburgher di carne bovina, 1 a carne macinata ed 1 a prosciutto cotto; per $\beta$-lattoglobulina, 1 ad hamburger, 1 medaglione di carne ed 1 a polpetta di carne (Tabella 3). Se si prende in considerazione il luogo del prelievo, si evince che, per la quasi totalità delle non conformità, si tratta di prodotti preparati artigianalmente presso macellerie 0 supermercati.

Tabella 1. Campioni analizzati, suddivisi per tipologia di esame.

\begin{tabular}{lc} 
Tlipologia di same & Esami (n) \\
Ricerca $\beta$-blattoglobulina - ELISA & 105 \\
Ricerca ovoalbumina - ELISA & 103 \\
\hline Totale & 208 \\
\hline
\end{tabular}


Tabella 2. Conformità dei campioni analizzati, suddivisi per tipologia di matrice ed esami effettuati.

\begin{tabular}{|c|c|c|c|c|c|c|}
\hline \multirow[t]{2}{*}{ Matrici } & \multirow[t]{2}{*}{$\begin{array}{l}\text { Numero } \\
\text { campioni }\end{array}$} & \multicolumn{2}{|c|}{$\begin{array}{l}\text { Ricerca ovoalbumina } \\
\text { ELISA }\end{array}$} & \multicolumn{2}{|c|}{$\begin{array}{c}\text { Ricerca } \beta \text {-lattoglobulina } \\
\text { ELISA }\end{array}$} & \multirow[t]{2}{*}{ Totale } \\
\hline & & Conforme & Non conforme & Conforme & Non conforme & \\
\hline Alimenti prima infanzia a base di carne & 2 & 1 & 0 & 2 & 0 & 3 \\
\hline $\begin{array}{l}\text { Alimenti destinati ad un'alimentazione particolare } \\
\text { non a base di prodotti di origine animale }\end{array}$ & 31 & 31 & 0 & 31 & 0 & 62 \\
\hline Carni macinate o preparazione di carni & 36 & 30 & 6 & 32 & 3 & 71 \\
\hline Omogeneizzati di carne & 16 & 16 & 0 & 15 & 0 & 31 \\
\hline Piatti pronti non a base di prodotti di origine animale & 22 & 19 & 0 & 22 & 0 & 41 \\
\hline Totale & 107 & 97 & 6 & 102 & 3 & 208 \\
\hline
\end{tabular}

Tabella 3. Dettaglio delle matrici alimentari e relative non conformità, comprensivo del luogo del prelievo.

\begin{tabular}{lcccc} 
Matrice & Luogo del prelievo & Ricerca ovoalbumina (n) & Ricerca betalattoglobulina (n) & Totale \\
Hamburger di carne bovina & Macelleria & 1 & 1 & 2 \\
Carne macinata & Mensa scolastica & 1 & 3 & 1 \\
\hline Salsiccia di suino & Macelleria & & 1 & 1 \\
Medaglione di carne si suino & Supermercato - banco frigo & 1 & 1 \\
\hline Polpetta di carne bovina & Supermercato - banco frigo & Supermercato - banco frigo & 1 & 1 \\
Prosciutto cotto & & & 1 & 1 \\
\hline
\end{tabular}

\section{Discussione e Conclusioni}

Dall'analisi dei dati si evince che i prodotti più a rischio allergeni sono quelli a base di carne, prodotti artigianalmente (Fragassi et al., 2008). L'evoluzione normativa nel settore degli allergeni, ha portato le aziende a prendere in considerazione, nei piani di autocontrollo, il pericolo allergeni come un rischio da gestire (Ciarrocchi et al., 2011). La gestione di tale rischio è peraltro complessa, vista la natura di tali sostanze, che non consente di attuare delle efficaci procedure di decontaminazione. Nella valutazione del rischio pertanto è necessario attuare un programma di controllo e gestione degli allergeni che preveda tutta una serie di attività quali: i) evitare incrocio dei flussi produttivi e del personale tra aree a rischio allergeni e aree non a rischio; ii) dedicare le attrezzature a linee con e senza allergeni 0 , se non possibile, procedere con produzione prima del prodotto senza allergene, prevedendo procedure di pulizia e sanificazione validate; iii) prevedere personale dedicato alla produzione di prodotti senza allergeni o, ove non possibile, prevedre cambio divisa, lavaggio mani e guanti; iv) contenere la contaminazione aerea in corrispondenza delle fasi in cui vengono impiegati ingredienti allergenici; v) controllo della materia prima, attraverso l'utilizzo di fornitori qualificati, in grado di garantire la fornitura di prodotti esenti da allergeni.

Dai dati ottenuti, si evince come la problema- tica allergeni venga gestita dalla maggior parte delle aziende secondo il principio della valutazione del rischio; le non conformità sono state rilevate nelle strutture di piccole dimensioni, che evidentemente non gestiscono sempre altrettanto efficacemente tale problematica.

\section{Bibliografia}

Bush RK, Hefle SL, 1996. Food allergens. Crc Cr Rev Food Sci 36:119-63.

Ciarrocchi M, Boniglia C, Giammarioli S, Sanzini E, 2011. Tutela dei consumatori affetti da allergie alimentari: presupposti e modalità di intervento. Istituto Superiore di Sanità, Roma.

Commissione Europea, 2003. Direttiva del Parlamento Europeo del Consiglio del 10 novembre 2003 che modifica la direttiva 2000/13/CE per quanto riguarda l'indicazione degli ingredienti contenuti nei prodotti alimentari, 2003/89/CE. In: Gazzetta Ufficiale, L 308/15, 25/11/2003.

Fragassi S, Lai J, Fabbri M, Adriano D, Gallina S, Bianchi DM, Barbaro A, Decastelli L, 2008. Determinazione di allergeni in prodotti a base di carne: monitoraggio in Piemonte nell'anno 2007. In: Atti X Congresso Nazionale S.I.Di.L.V., Alghero 22-24 ottobre 2008, pp 40-41.

Madsen CH, 2005. Prevalence of food allergy: an overview. P Nutr Soc 64:413-7.

Osterballe M, Hansen TK, Mortz CG, BindslevJensen C, 2005. The clinical relevance of sensitization to pollen-related fruits and vegetables in unselected pollen-sensitized adults. Allergy 60:218-25.

Regione Campania, 2011. Piano regionale integrato dei controlli ufficiali in materia di alimenti, mangimi, sanità e benessere animale e sanità delle piante (P.R.I.) 20112014, DGRG 377/11. Disponibile al sito: http://agricoltura.regione.campania.it/com unicati/pdf/DGR_377-04-08-11.pdf

Repubblica Italiana, 2006. Decreto legislativo n. 114 dell'8 febbraio 2006. Attuazione delle direttive 2003/89/CE, 2004/77/CE e 2005/63/CE in materia di indicazione degli ingredienti contenuti nei prodotti alimentari. In: Gazzetta Ufficiale n. 69, 23 marzo 2006.

Roehr CC, Edenharter G, Reimann S, Ehlers I, Worm M, Zuberbier T, Niggemann B, 2004. Food allergy and non-allergic food hypersensitivity in children and adolescents. Clin Exp Allergy 34:1534-41.

Taylor SL, Hefle SL, Munoz-Furlong A, 1999. Food allergies and avoidance diets. Nutr Today 34:15-22.

Zuberbier T, Edenharter G, Worm M, Ehlers I, Reimann S, Hantke T, Roehr CC, Bergmann KE, Niggemann B, 2004. Prevalence of adverse reactions to food in Germany: a population study. Allergy 59:338-45. 